\title{
Impact of Different Graded Levels of Fertilizers on the Incidence of Yellow Stem Borer Scirpophaga incertulas (Wlk.), Leaf Folder Cnaphalocrocis medinalis (Guenee) and Whorl Maggot Hydrellia philippina (Ferino) in Paddy
}

\author{
G. Madhuri*, P.C. Dash and P. Pruthvi \\ Department of Entomology, College of Agriculture, Orissa University of Agriculture and \\ Technology, Bhubaneswar, India \\ *Corresponding author
}

\begin{tabular}{|c|c|}
\hline & A B S T R A C T \\
\hline & The field experiment was conducted at Central Research Farm of Orissa \\
\hline Keywords & University of Agriculture and Technology, Bhubaneswar during summer \\
\hline $\begin{array}{l}\text { Paddy, Yellow } \\
\text { Stem Borer, } \\
\text { Leaf folder, } \\
\text { NPK, Manures. }\end{array}$ & $\begin{array}{l}\text { 2014-15 and kharif- } 2015 \text { to evaluate the effect of different graded levels of } \\
\text { fertilizers and manure on incidence of yellow stem borer, leaf folder and } \\
\text { whorl maggot in paddy with twelve treatments and four replications in sub } \\
\text { plot size of } 15 \mathrm{~m} \times 10 \mathrm{~m} \text { taking Lalat and Swarna as test cultivar during }\end{array}$ \\
\hline Article Info & summer $2014-15$ and kharif -2015 following the recommended package of \\
\hline $\begin{array}{l}\text { Accepted: } \\
\text { 14 July } 2017 \\
\text { Available Online: } \\
\text { 10 September } 2017\end{array}$ & $\begin{array}{l}\text { practices It was observed that the incidence of borer, leaf folder and whorl } \\
\text { maggot was enhanced with application of increased doses of NPK } \\
\text { fertilizers. But the FYM and micronutrients with NPK nutrients recorded } \\
\text { low incidence of borer both at vegetative (dead heart) and heading stage } \\
\text { (white ear head), leaf folder and whorl maggot. }\end{array}$ \\
\hline
\end{tabular}

\section{Introduction}

Rice (Oryza sativa L.) is an important staple food crop for more than half of the world population and accounts for more than $50 \%$ of the daily calorie intake (Khush, 2005). Insect pests and diseases pose a very serious challenge in improving the productivity and achieving sustainability. Approximately 52\% of the global production of rice is lost annually owing to the damage caused by biotic stress factors, of which $21 \%$ is attributed to the attack of insect pests (Yarasi et al., 2008). The overall losses due to insect damage in rice were estimated to be 25 per cent (Dhaliwal et al., 2010). Out of this, 20 to 30 per cent damage is alone done by yellow stem borer, Scirpophaga incertulas (Walker) (Lal, 1996). However, Singh et al., (2003) reported $12-18.8 \%$ yield loss due to leaf folder in irrigated rice crop. High intensity of insect pest menace coupled with inadequate and imbalanced plant nutrient use is considered as major factors of low productivity. Judicious use of NPK fertilizers is considered as vital cultural practices in the IPM strategy of rice which minimise the insect pest incidence, markedly increase the 
yield and improve the quality of rice. Nitrogen, phosphorus and potassium fertilizers are major essential plant nutrients of rice production. Excessive application of nitrogenous fertilizers increased the incidence of insect pests by altering morphological, biochemical and physiological characters of host plants through host selection and ecological fitness such as survival, growth, fecundity and significant reduction of host resistance against herbivores improving the nutritional conditions for herbivores (Simpson, 1990, Barbour, 1991). Phosphorus is an important component for the population growth of phytophagous insects as it is required for RNA synthesis. On the contrary, application of potassium $(\mathrm{K})$ as a major plant nutrient enhances the protein synthesis and thus, reducing amino acid content in the sap there by making the plant less favourable for the reproduction of sucking pests (Samiyyan and Janathanan, 1988). Thus, fertilization not only influences the growth, development and yield of crop plants but also regulate the activities of insect pests both directly and indirectly with ultimate impact on yield. In this context, the economic doses of given nutrient, however, is to be determined critically considering its rate as plant nutrient and insect suppressant or promoter.

\section{Materials and Methods}

The field experiments were laid out during summer 2014-15 and kharif 2015 Randomized block design (RBD) with twelve treatments and four replications at Central Farm, OUAT, Bhubaneswar to assess the incidence of yellow stem borer (YSB), leaf folder (LF) and whorl maggot (WM) of rice raised under NPK nutrient levels with FYM and micro nutrients. The soil of the experimental plot was lateritic sandy loam.

The seedlings of rice variety Lalat and Swarna was taken as the test cultivar in summer 2014-15 and Kharif-2015 were planted in plots of size $15 \mathrm{~m} \times 10 \mathrm{~m}$ at spacing of $20 \mathrm{~cm} \times 10 \mathrm{~cm}$ with recommended agronomic practices.

\section{Methods and time of application of manures and fertilizers}

Nitrogen was applied in 3 splits i.e. $25 \%$ as basal, $50 \%$ at 15 days after transplanting at maximum tillering stage and $25 \%$ at panicle initiation stage. Total phosphorus and sulphur was applied as basal. Potash was applied as $50 \%$ basal and $50 \%$ at panicle initiation stage.

Boron was applied as two foliar sprays at $0.25 \%$ at panicle initiation stage and 15 days after panicle initiation. The required amount of FYM was incorporated one week before transplanting.

\section{Sampling techniques for record of pest population}

\section{Stem borer}

In vegetative stage, the total number of tillers and the dead hearts (DH) was counted in randomly selected 10 hills per subplot in replication by leaving the border lines from sides. The percentage of DH was computed from 30 DAT onwards up to 50 DAT during summer 2014-15 and up to 60DAT during Kharif 2015. At pre-harvest stage i.e., 15 days prior to harvest, the stem borer damage was assessed by computing white ear head percentage as suggested by (Gomez, 1972).

\section{Leaf folder}

Total number of leaves and the number of damaged leaves were counted in 10 randomly selected clumps of each subplot replication wise and the percentage leaf damage was computed from 30 DAT onwards up to 80 DAT during both the seasons. 


\section{Whorl maggot}

In vegetative stage, the total number of tillers and the damaged leaves were counted in 10 randomly selected hills/subplot in each replication wise and the percentage leaf damage was computed from 30 DAT onwards up to 50 DAT during both the seasons.

The data thus obtained on extent of damage by stem borer (DH and WEH \%), leaf folder (\% leaf damage) and whorl maggot (\% leaf damage) were computed and the mean data were subjected to statistical analysis after suitable transformation.

\section{Results and Discussion}

Effect of fertilizers, fertilizer combinations and manure on incidence of yellow stem borer

The data on incidence of stem borer (\% DH \& $\%$ WEH) during summer 2014-15 is presented in table 1 , it was observed that mean \% DH was lowest in $\mathrm{T}_{5}(100 \% \mathrm{NPK}+$ FYM) followed by $\mathrm{T}_{4}(100 \% \mathrm{NPK}+\mathrm{Zn}), \mathrm{T}_{7}$ $(100 \% \mathrm{NPK}+\mathrm{B}+\mathrm{Zn})$ and $\mathrm{T}_{6}(100 \% \mathrm{NPK}$ $+\mathrm{FYM}+$ Lime) then rest of the treatments in which the mean \% DH more than $15 \%$.Thus, it clearly indicated that individual nutrient or combination of nutrients i.e. NPK produced more DH has compared to $100 \%$ NPK supplemented with either B, Zn, \& FYM that supported very few adult population of YSB. During kharif 2015 there was a change in trend as regards to $\% \mathrm{DH}$, the treatment $\mathrm{T}_{7}$ $(100 \% \mathrm{NPK}+\mathrm{B}+\mathrm{Zn})$ produced only mean of $8.45 \% \mathrm{DH}$ followed by $\mathrm{T}_{4}(100 \% \mathrm{NPK}$ $+\mathrm{Zn}), \mathrm{T}_{8}(100 \% \mathrm{NPK}+\mathrm{S}+\mathrm{Zn}), \mathrm{T}_{6}(100 \%$ $\mathrm{NPK}+\mathrm{FYM}+\mathrm{Lime}), \mathrm{T}_{5}(100 \% \mathrm{NPK}+\mathrm{FYM})$ (Table 2). It is very clear that irrespective of season the treatments comprising $100 \% \mathrm{NPK}$ with supplemented with $\mathrm{Zn}, \mathrm{B}$ and $\mathrm{S}$ produced less DH as compared to rest of the treatments. This gives a clear cut idea that a constituent of FYM with balanced nutrition was responsible for low $\mathrm{DH}$, which has been earlier observed by Chakraborty et al., (2011). Shahjahan (1992) concluded that the infestation of yellow stem borer decreased with increase of dosage of zinc sulphate. Dash et al., (2008) observed that supplementation of $\mathrm{ZnSO}_{4}$ with NPK nutrients recorded low incidence of borer both at vegetative (DH) and heading stage (WEH). Sarwar (2011) and Dash et al., (2011) observed decreased yellow stem borer infestation with supplementation of $\mathrm{ZnSO}_{4}$. Pandal et al., (1975) and Panda (1976) reported the antagonistic effect of zinc against yellow stem borer which resulted due to induced antibiosis effect of zinc and developing of hard pseudostem. Shu et al., (2009) reported excess $\mathrm{Zn}$ made expression of vitellogenin gene down regulated and caused poor accumulation of egg yolk, which led to reduction in egg numbers and failure of eggs to hatch. Thus, the present findings derived ample support from the finding of above workers, so far the dead heart and white ear head was concerned.

\section{Effect of fertilizers, fertilizer combinations and manure on incidence of leaf folder}

The data on effect of NPK fertilizer alone or in different combination along with supplement with lime, FYM, B, S, Zn etc on the incidence of leaf folder in summer 201415 and kharif 2015 have been present in tables 3 and 4 respectively. It was observed that during summer season $\mathrm{T}_{6}(100 \% \mathrm{NPK}+$ FYM + Lime) produced lowest minimum leaf damage of $3.13 \%$, followed by $\mathrm{T}_{5}(100 \%$ $\mathrm{NPK}+\mathrm{FYM}), \mathrm{T}_{7}(100 \% \mathrm{NPK}+\mathrm{B}+\mathrm{Zn}), \mathrm{T}_{2}$ $(100 \% \mathrm{NPK})$ and $\mathrm{T}_{1}(100 \% \mathrm{PK})$ treatments respectively. Whereas, in kharif- 2015 leaf damage found to be little bit high being lowest in $\mathrm{T}_{7}(100 \% \mathrm{NPK}+\mathrm{B}+\mathrm{Zn})$ followed 
by $\mathrm{T}_{4}, \mathrm{~T}_{8}, \mathrm{~T}_{11}$ etc. The treatment $\mathrm{T}_{7}$ consistently produced less damage by leaf folder during both years of study, which clearly indicated that $100 \%$ NPK with B and $\mathrm{Zn}$ accounted for least leaf folder damage. Chau et al., (2005) reported reduction of 50$60 \%$ incidence of leaf folder on application of FYM at dosage of $5.0-7.5 \mathrm{t} \mathrm{ha}^{-1}$. Plants sprayed with boron and zinc chelates lowered the incidence of leaf folder as earlier reported by Premila et al., (1984). Hyrsl et al., (2007) reported that boric acid has adverse effect on survival, development and fecundity of insects. Hence, the present finding is well supported by above findings.

Table.1 Incidence of yellow stem borer (DH \%) and (WEH \%) in rice during summer 2014-2015, at Bhubaneswar

\begin{tabular}{|c|c|c|c|c|c|}
\hline \multirow[t]{2}{*}{ Treatments } & \multicolumn{3}{|c|}{ Incidence of DH (\%) at } & \multirow{2}{*}{$\begin{array}{c}\text { Mean } \\
\text { (\%) DH }\end{array}$} & \multirow[t]{2}{*}{ WEH (\%) } \\
\hline & 30 DAT & 40DAT & 50DAT & & \\
\hline $\mathrm{T}_{1}-100 \% \mathrm{PK}$ & $\begin{array}{l}20.96 \\
(4.54)\end{array}$ & $\begin{array}{l}21.92 \\
(4.72)\end{array}$ & $\begin{array}{l}22.30 \\
(4.76)\end{array}$ & 21.72 & $\begin{array}{l}8.15 \\
(2.99)\end{array}$ \\
\hline $\mathrm{T} \mathrm{T}_{2}-100 \% \mathrm{NPK}$ & $\begin{array}{l}19.15 \\
(4.42)\end{array}$ & $\begin{array}{l}20.77 \\
(4.60)\end{array}$ & $\begin{array}{l}21.90 \\
(4.72)\end{array}$ & 20.60 & $\begin{array}{l}7.35 \\
(2.64)\end{array}$ \\
\hline $\mathrm{T}_{3}-150 \% \mathrm{NPK}$ & $\begin{array}{l}20.94 \\
(4.70)\end{array}$ & $\begin{array}{l}21.42 \\
(4.67)\end{array}$ & $\begin{array}{l}22.20 \\
(4.76)\end{array}$ & 21.52 & $\begin{array}{l}7.93 \\
(2.89)\end{array}$ \\
\hline $\mathrm{T}_{4}-100 \% \mathrm{NPK}+\mathrm{Zn}$ & $\begin{array}{l}10.33 \\
(3.20)\end{array}$ & $\begin{array}{l}11.46 \\
(3.38)\end{array}$ & $\begin{array}{l}12.45 \\
(3.52)\end{array}$ & 11.41 & $\begin{array}{l}6.67 \\
(2.67)\end{array}$ \\
\hline $\mathrm{T}_{5}-100 \% \mathrm{NPK}+\mathrm{FYM}$ & $\begin{array}{l}9.10 \\
(3.13)\end{array}$ & $\begin{array}{l}10.73 \\
(3.27)\end{array}$ & $\begin{array}{l}11.63 \\
(3.40)\end{array}$ & 10.73 & $\begin{array}{l}6.26 \\
(2.59)\end{array}$ \\
\hline $\begin{array}{l}\mathrm{T}_{6}-100 \% \mathrm{NPK}+\mathrm{FYM}+ \\
\text { Lime }\end{array}$ & $\begin{array}{l}12.49 \\
(3.59)\end{array}$ & $\begin{array}{l}13.85 \\
(3.78)\end{array}$ & $\begin{array}{l}14.46 \\
(3.86)\end{array}$ & 13.60 & $\begin{array}{l}5.30 \\
(2.41)\end{array}$ \\
\hline $\mathrm{T}_{7}-100 \% \mathrm{NPK}+\mathrm{B}+\mathrm{Zn}$ & $\begin{array}{l}11.03 \\
(3.31)\end{array}$ & $\begin{array}{l}12.03 \\
(3.46)\end{array}$ & $\begin{array}{l}13.03 \\
(3.60)\end{array}$ & 12.03 & $\begin{array}{l}6.67 \\
(2.67)\end{array}$ \\
\hline $\mathrm{T}_{8}-100 \% \mathrm{NPK}+\mathrm{S}+\mathrm{Zn}$ & $\begin{array}{l}16.38 \\
(4.10)\end{array}$ & $\begin{array}{l}17.85 \\
(4.27)\end{array}$ & $\begin{array}{l}18.70 \\
(4.34)\end{array}$ & 17.64 & $\begin{array}{l}6.46 \\
(2.61)\end{array}$ \\
\hline $\mathrm{T}_{9}-100 \% \mathrm{~N}$ & $\begin{array}{l}24.23 \\
(4.96)\end{array}$ & $\begin{array}{l}24.95 \\
(5.04)\end{array}$ & $\begin{array}{l}28.40 \\
(4.84)\end{array}$ & 25.86 & $\begin{array}{l}11.82 \\
(3.50)\end{array}$ \\
\hline $\mathrm{T}_{10}-100 \% \mathrm{NP}$ & $\begin{array}{l}21.33 \\
(4.62)\end{array}$ & $\begin{array}{l}22.67 \\
(4.80)\end{array}$ & $\begin{array}{l}23.00 \\
(4.84)\end{array}$ & 22.33 & $\begin{array}{l}9.75 \\
(3.19)\end{array}$ \\
\hline $\mathrm{T}_{11}-100 \% \mathrm{NPK}+$ Lime & $\begin{array}{l}17.05 \\
(4.35)\end{array}$ & $\begin{array}{l}18.91 \\
(4.59)\end{array}$ & $\begin{array}{l}20.45 \\
(4.59)\end{array}$ & 18.80 & $\begin{array}{l}6.95 \\
(2.71)\end{array}$ \\
\hline $\mathrm{T}_{12}$ - Control & $\begin{array}{l}25.90 \\
(5.13)\end{array}$ & $\begin{array}{l}27.12 \\
(5.25)\end{array}$ & $\begin{array}{l}29.27 \\
(5.48)\end{array}$ & 27.43 & $\begin{array}{l}14.40 \\
(3.85)\end{array}$ \\
\hline $\mathrm{SE}(\mathrm{m}) \pm$ & 0.06 & 0.06 & 0.32 & - & 0.07 \\
\hline $\mathrm{CD}(5 \%)$ & 0.19 & 0.17 & 0.92 & - & 0.21 \\
\hline $\mathrm{CV}(\%)$ & 3.10 & 2.78 & 14.44 & - & 5.01 \\
\hline
\end{tabular}

Figures in parenthesis are $\sqrt{(\mathrm{x}+0.5)}$ transformed values. $100 \% \mathrm{NPK}=80: 40: 60 \mathrm{~kg}$ ha $1\left(\mathrm{~N}^{-}: \mathrm{P}_{2} \mathrm{O}_{5}: \mathrm{K}_{2} \mathrm{O}\right) ; \mathrm{FYM}=5 \mathrm{t}$ $\mathrm{ha}^{-1} ;$ Lime $=1 \mathrm{t} \mathrm{ha}^{-1 ;} \mathrm{Zn}=12.5 \mathrm{~kg} \mathrm{Zn} \mathrm{SO}_{4} \mathrm{ha}^{-1 ;} \mathrm{B}=0.25 \% ; \mathrm{S}=30 \mathrm{~kg}_{\text {gypsum ha }}{ }^{-1}$. 
Table.2 Incidence of yellow stem borer (DH \%) and (WEH \%) in rice during kharif-2015, at Bhubaneswar

\begin{tabular}{|c|c|c|c|c|c|c|}
\hline \multirow[t]{2}{*}{ Treatments } & \multicolumn{4}{|c|}{ Incidence of DH (\%) at } & \multirow[t]{2}{*}{ Mean (\% DH) } & \multirow[t]{2}{*}{ WEH (\%) } \\
\hline & 30 DAT & 40DAT & 50DAT & 60DAT & & \\
\hline $\mathrm{T}_{1}-100 \% \mathrm{PK}$ & $\begin{array}{l}11.21 \\
(3.41)\end{array}$ & $\begin{array}{l}12.40 \\
(3.58)\end{array}$ & $\begin{array}{l}12.87 \\
(3.62)\end{array}$ & $\begin{array}{l}13.45 \\
(3.73)\end{array}$ & 12.48 & $\begin{array}{l}4.12 \\
(2.13)\end{array}$ \\
\hline $\mathrm{T} \mathrm{T}_{2}-100 \% \mathrm{NPK}$ & $\begin{array}{l}10.32 \\
(3.25)\end{array}$ & $\begin{array}{l}11.07 \\
(3.39)\end{array}$ & $\begin{array}{l}11.27 \\
(3.42)\end{array}$ & $\begin{array}{l}11.67 \\
(3.48)\end{array}$ & 11.08 & $\begin{array}{l}3.62 \\
(2.02)\end{array}$ \\
\hline $\mathrm{T}_{3}-150 \% \mathrm{NPK}$ & $\begin{array}{l}11.21 \\
(3.41)\end{array}$ & $\begin{array}{l}12.17 \\
(3.55)\end{array}$ & $\begin{array}{l}12.50 \\
(3.60)\end{array}$ & $\begin{array}{l}12.70 \\
(3.62)\end{array}$ & 12.14 & $\begin{array}{l}3.85 \\
(2.08)\end{array}$ \\
\hline $\mathrm{T}_{4}-100 \% \mathrm{NPK}+\mathrm{Zn}$ & $\begin{array}{l}7.62 \\
(2.84)\end{array}$ & $\begin{array}{l}9.20 \\
(3.11)\end{array}$ & $\begin{array}{l}9.40 \\
(3.13)\end{array}$ & $\begin{array}{l}10.35 \\
(3.28)\end{array}$ & 9.14 & $\begin{array}{l}2.92 \\
(1.84)\end{array}$ \\
\hline $\begin{array}{l}\mathrm{T}_{5}-100 \% \mathrm{NPK}+ \\
\text { FYM }\end{array}$ & $\begin{array}{l}9.59 \\
(3.16)\end{array}$ & $\begin{array}{l}10.70 \\
(3.34)\end{array}$ & $\begin{array}{l}10.67 \\
(3.33)\end{array}$ & $\begin{array}{l}10.90 \\
(3.36)\end{array}$ & 10.46 & $\begin{array}{l}3.42 \\
(1.96)\end{array}$ \\
\hline $\begin{array}{l}\mathrm{T}_{6}-100 \% \text { NPK }+\mathrm{FYM}+ \\
\text { Lime }\end{array}$ & $\begin{array}{l}9.09 \\
(3.09)\end{array}$ & $\begin{array}{l}10.50 \\
(3.31)\end{array}$ & $\begin{array}{l}10.15 \\
(3.17)\end{array}$ & $\begin{array}{l}9.77 \\
(3.20)\end{array}$ & 9.89 & $\begin{array}{l}3.22 \\
(1.92)\end{array}$ \\
\hline $\mathrm{T}_{7}-100 \% \mathrm{NPK}+\mathrm{B}+\mathrm{Zn}$ & $\begin{array}{l}7.42 \\
(2.79)\end{array}$ & $\begin{array}{l}8.42 \\
(2.98)\end{array}$ & $\begin{array}{l}8.62 \\
(3.01)\end{array}$ & $\begin{array}{l}9.35 \\
(3.13)\end{array}$ & 8.45 & $\begin{array}{l}2.90 \\
(1.81)\end{array}$ \\
\hline $\mathrm{T}_{8}-100 \% \mathrm{NPK}+\mathrm{S}+\mathrm{Zn}$ & $\begin{array}{l}8.79 \\
(2.96)\end{array}$ & $\begin{array}{l}9.57 \\
(3.12)\end{array}$ & $\begin{array}{l}9.50 \\
(3.37)\end{array}$ & $\begin{array}{l}10.45 \\
(3.30)\end{array}$ & 9.57 & $\begin{array}{l}3.02 \\
(1.86)\end{array}$ \\
\hline $\mathrm{T}_{9}-100 \% \mathrm{~N}$ & $\begin{array}{l}12.23 \\
(3.52)\end{array}$ & $\begin{array}{l}13.30 \\
(3.69)\end{array}$ & $\begin{array}{l}13.47 \\
(3.57)\end{array}$ & $\begin{array}{l}14.02 \\
(3.80)\end{array}$ & 13.25 & $\begin{array}{l}5.67 \\
(2.48)\end{array}$ \\
\hline $\mathrm{T}_{10}-100 \% \mathrm{NP}$ & $\begin{array}{l}11.79 \\
(3.53)\end{array}$ & $\begin{array}{l}13.30 \\
(3.71)\end{array}$ & $\begin{array}{l}13.35 \\
(3.71)\end{array}$ & $\begin{array}{l}13.87 \\
(3.78)\end{array}$ & 13.07 & $\begin{array}{l}4.42 \\
(2.21)\end{array}$ \\
\hline $\mathrm{T}_{11}-100 \% \mathrm{NPK}+$ Lime & $\begin{array}{l}9.73 \\
(3.13)\end{array}$ & $\begin{array}{l}10.80 \\
(3.33)\end{array}$ & $\begin{array}{l}10.45 \\
(3.30)\end{array}$ & $\begin{array}{l}10.52 \\
(3.31)\end{array}$ & 10.28 & $\begin{array}{l}3.37 \\
(1.82)\end{array}$ \\
\hline $\mathrm{T}_{12^{-}}$Control & $\begin{array}{l}14.36 \\
(3.86)\end{array}$ & $\begin{array}{l}15.30 \\
(3.97)\end{array}$ & $\begin{array}{l}15.30 \\
(3.97)\end{array}$ & $\begin{array}{l}15.50 \\
(3.99)\end{array}$ & 15.12 & $\begin{array}{l}7.42 \\
(2.80)\end{array}$ \\
\hline $\mathrm{SE}(\mathrm{m}) \pm$ & 0.09 & 0.09 & 0.10 & 0.07 & - & 0.08 \\
\hline $\mathrm{CD}(5 \%)$ & 0.26 & 0.25 & 0.29 & 0.20 & - & 0.24 \\
\hline $\mathrm{CV}(\%)$ & 5.54 & 4.98 & 5.78 & 4.00 & - & 7.89 \\
\hline
\end{tabular}

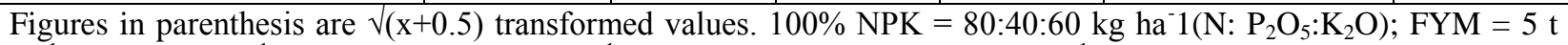
$\mathrm{ha}^{-1 ;}$ Lime $=1 \mathrm{t} \mathrm{ha}^{-1 ;} \mathrm{Zn}=12.5 \mathrm{~kg} \mathrm{Zn} \mathrm{SO}_{4} \mathrm{ha}^{-1 ;} \mathrm{B}=0.25 \% ; \mathrm{S}=30 \mathrm{~kg}_{\text {gypsum ha }}{ }^{-1}$. 
Table.3 Incidence of leaf folder in rice during summer 2014-2015, at Bhubaneswar

\begin{tabular}{|c|c|c|c|c|c|}
\hline \multirow[t]{2}{*}{ Treatments } & \multicolumn{4}{|c|}{ Incidence of LF (\%) at } & \multirow{2}{*}{$\begin{array}{l}\text { Mean (\%) } \\
\text { leaf damage }\end{array}$} \\
\hline & 50 DAT & 60DAT & 70DAT & 80DAT & \\
\hline $\mathrm{T}_{1}-100 \% \mathrm{PK}$ & $\begin{array}{l}3.07 \\
(1.87)\end{array}$ & $\begin{array}{l}3.62 \\
(2.03)\end{array}$ & $\begin{array}{l}3.60 \\
(2.21)\end{array}$ & $\begin{array}{l}4.05 \\
(2.13)\end{array}$ & 3.58 \\
\hline $\mathrm{T} \mathrm{T}_{2}-100 \% \mathrm{NPK}$ & $\begin{array}{l}2.87 \\
(1.80)\end{array}$ & $\begin{array}{l}3.07 \\
(1.88)\end{array}$ & $\begin{array}{l}3.95 \\
(2.10)\end{array}$ & $\begin{array}{l}4.42 \\
(2.22)\end{array}$ & 3.57 \\
\hline $\mathrm{T}_{3}-150 \% \mathrm{NPK}$ & $\begin{array}{l}5.32 \\
(2.40)\end{array}$ & $\begin{array}{l}6.22 \\
(2.63)\end{array}$ & $\begin{array}{l}6.72 \\
(2.67)\end{array}$ & $\begin{array}{l}7.55 \\
(2.83)\end{array}$ & 6.45 \\
\hline $\mathrm{T}_{4}-100 \% \mathrm{NPK}+\mathrm{Zn}$ & $\begin{array}{l}4.07 \\
(2.13)\end{array}$ & $\begin{array}{l}5.02 \\
(2.29)\end{array}$ & $\begin{array}{l}5.70 \\
(2.48)\end{array}$ & $\begin{array}{l}6.90 \\
(2.72)\end{array}$ & 5.42 \\
\hline $\mathrm{T}_{5}-100 \% \mathrm{NPK}+\mathrm{FYM}$ & $\begin{array}{l}2.50 \\
(1.74)\end{array}$ & $\begin{array}{l}2.57 \\
(1.75)\end{array}$ & $\begin{array}{l}4.72 \\
(2.26)\end{array}$ & $\begin{array}{l}3.77 \\
(2.32)\end{array}$ & 3.39 \\
\hline $\begin{array}{l}\mathrm{T}_{6}-100 \% \text { NPK+ FYM + } \\
\text { Lime }\end{array}$ & $\begin{array}{l}2.55 \\
(1.74)\end{array}$ & $\begin{array}{l}2.72 \\
(1.79)\end{array}$ & $\begin{array}{l}3.47 \\
(1.99)\end{array}$ & $\begin{array}{l}3.80 \\
(1.87)\end{array}$ & 3.13 \\
\hline $\mathrm{T}_{7}-100 \% \mathrm{NPK}+\mathrm{B}+\mathrm{Zn}$ & $\begin{array}{l}2.65 \\
(1.76)\end{array}$ & $\begin{array}{l}2.97 \\
(1.86)\end{array}$ & $\begin{array}{l}3.77 \\
(2.01)\end{array}$ & $\begin{array}{l}4.22 \\
(2.17)\end{array}$ & 3.40 \\
\hline $\mathrm{T}_{8}-100 \% \mathrm{NPK}+\mathrm{S}+\mathrm{Zn}$ & $\begin{array}{l}3.07 \\
(1.88)\end{array}$ & $\begin{array}{l}4.37 \\
(2.20)\end{array}$ & $\begin{array}{l}4.87 \\
(2.31)\end{array}$ & $\begin{array}{l}5.70 \\
(2.48)\end{array}$ & 4.50 \\
\hline $\mathrm{T}_{9}-100 \% \mathrm{~N}$ & $\begin{array}{l}5.60 \\
(2.45)\end{array}$ & $\begin{array}{l}6.80 \\
(2.69)\end{array}$ & $\begin{array}{l}7.05 \\
(2.72)\end{array}$ & $\begin{array}{l}8.45 \\
(2.98)\end{array}$ & 6.97 \\
\hline $\mathrm{T}_{10}-100 \% \mathrm{NP}$ & $\begin{array}{l}5.47 \\
(2.30)\end{array}$ & $\begin{array}{l}6.60 \\
(2.65)\end{array}$ & $\begin{array}{l}7.40 \\
(2.81)\end{array}$ & $\begin{array}{l}7.82 \\
(2.88)\end{array}$ & 6.82 \\
\hline $\mathrm{T}_{11}-100 \% \mathrm{NPK}+$ Lime & $\begin{array}{l}2.80 \\
(1.83)\end{array}$ & $\begin{array}{l}3.17 \\
(2.08)\end{array}$ & $\begin{array}{l}3.92 \\
(2.32)\end{array}$ & $\begin{array}{l}4.35 \\
(2.29)\end{array}$ & 3.56 \\
\hline $\mathrm{T}_{12-}$ Control & $\begin{array}{l}5.00 \\
(2.34)\end{array}$ & $\begin{array}{l}6.00 \\
(2.55)\end{array}$ & $\begin{array}{l}6.25 \\
(2.54)\end{array}$ & $\begin{array}{l}7.15 \\
(2.71)\end{array}$ & 6.10 \\
\hline $\mathrm{SE}(\mathrm{m}) \pm$ & 0.08 & 0.08 & 0.10 & 0.05 & - \\
\hline $\mathrm{CD}(5 \%)$ & 0.25 & 0.25 & 0.29 & 0.16 & - \\
\hline CV (\%) & 8.86 & 8.07 & 8.70 & 4.76 & - \\
\hline
\end{tabular}

Figures in parenthesis are $\sqrt{ }(\mathrm{x}+0.5)$ transformed values. $100 \% \mathrm{NPK}=80: 40: 60 \mathrm{~kg}$ ha ${ }^{-} 1\left(\mathrm{~N}: \mathrm{P}_{2} \mathrm{O}_{5}: \mathrm{K}_{2} \mathrm{O}\right) ; \mathrm{FYM}=5 \mathrm{t}$ $\mathrm{ha}^{-1 ;}$ Lime $=1 \mathrm{t} \mathrm{ha}^{-1 ;} \mathrm{Zn}=12.5 \mathrm{~kg} \mathrm{Zn} \mathrm{SO}_{4} \mathrm{ha}^{-1 ;} \mathrm{B}=0.25 \% ; \mathrm{S}=30 \mathrm{~kg}_{\text {gypsum ha }}{ }^{-1}$. 
Table.4 Incidence of leaf folder in rice during kharif-2015, at Bhubaneswar

\begin{tabular}{|c|c|c|c|c|c|}
\hline \multirow[t]{2}{*}{ Treatments } & \multicolumn{4}{|c|}{ Leaf damage (\%) at } & \multirow{2}{*}{$\begin{array}{l}\text { Mean(\%) lea } \\
\text { damage }\end{array}$} \\
\hline & 50 DAT & 60DAT & 70DAT & 80DAT & \\
\hline $\mathrm{T}_{1}-100 \% \mathrm{PK}$ & $\begin{array}{l}8.15 \\
(2.92)\end{array}$ & $\begin{array}{l}7.95 \\
(2.89)\end{array}$ & $\begin{array}{l}8.14 \\
(2.92)\end{array}$ & $\begin{array}{l}10.61 \\
(3.32)\end{array}$ & 8.71 \\
\hline $\mathrm{T} \mathrm{T}_{2}-100 \% \mathrm{NPK}$ & $\begin{array}{l}6.15 \\
(2.56)\end{array}$ & $\begin{array}{l}7.03 \\
(2.70)\end{array}$ & $\begin{array}{l}6.96 \\
(2.72)\end{array}$ & $\begin{array}{l}6.80 \\
(2.69)\end{array}$ & 6.73 \\
\hline $\mathrm{T}_{3}-150 \% \mathrm{NPK}$ & $\begin{array}{l}6.15 \\
(2.54)\end{array}$ & $\begin{array}{l}7.28 \\
(2.78)\end{array}$ & $\begin{array}{l}7.03 \\
(2.70)\end{array}$ & $\begin{array}{l}8.76 \\
(3.03)\end{array}$ & 7.30 \\
\hline $\mathrm{T}_{4}-100 \% \mathrm{NPK}+\mathrm{Zn}$ & $\begin{array}{l}5.10 \\
(2.35)\end{array}$ & $\begin{array}{l}4.53 \\
(2.24)\end{array}$ & $\begin{array}{l}5.06 \\
(2.35)\end{array}$ & $\begin{array}{l}4.68 \\
(2.27)\end{array}$ & 4.84 \\
\hline $\mathrm{T}_{5}-100 \% \mathrm{NPK}+\mathrm{FYM}$ & $\begin{array}{l}5.75 \\
(2.49)\end{array}$ & $\begin{array}{l}6.03 \\
(2.55)\end{array}$ & $\begin{array}{l}6.74 \\
(2.65)\end{array}$ & $\begin{array}{l}6.68 \\
(2.67)\end{array}$ & 6.30 \\
\hline $\mathrm{T}_{6}-100 \% \mathrm{NPK}+\mathrm{FYM}+$ Lime & $\begin{array}{l}5.50 \\
(2.44)\end{array}$ & $\begin{array}{l}5.12 \\
(2.35)\end{array}$ & $\begin{array}{l}5.64 \\
(2.46)\end{array}$ & $\begin{array}{l}6.22 \\
(2.58)\end{array}$ & 8.62 \\
\hline $\mathrm{T}_{7}-100 \% \mathrm{NPK}+\mathrm{B}+\mathrm{Zn}$ & $\begin{array}{l}5.10 \\
(2.34)\end{array}$ & $\begin{array}{l}4.29 \\
(2.18)\end{array}$ & $\begin{array}{l}4.96 \\
(2.33)\end{array}$ & $\begin{array}{l}4.61 \\
(2.25)\end{array}$ & 4.74 \\
\hline $\mathrm{T}_{8}-100 \% \mathrm{NPK}+\mathrm{S}+\mathrm{Zn}$ & $\begin{array}{l}5.30 \\
(2.38)\end{array}$ & $\begin{array}{l}4.95 \\
(2.33)\end{array}$ & $\begin{array}{l}5.56 \\
(2.45)\end{array}$ & $\begin{array}{l}5.99 \\
(2.54)\end{array}$ & 5.45 \\
\hline $\mathrm{T}_{9}-100 \% \mathrm{~N}$ & $\begin{array}{l}10.90 \\
(3.35)\end{array}$ & $\begin{array}{l}11.33 \\
(3.42)\end{array}$ & $\begin{array}{l}10.56 \\
(3.32)\end{array}$ & $\begin{array}{l}12.99 \\
(3.67)\end{array}$ & 11.44 \\
\hline $\mathrm{T}_{10}-100 \% \mathrm{NP}$ & $\begin{array}{l}9.95 \\
(3.20)\end{array}$ & $\begin{array}{l}10.78 \\
(3.28)\end{array}$ & $\begin{array}{l}9.60 \\
(3.16)\end{array}$ & $\begin{array}{l}11.61 \\
(3.47)\end{array}$ & 10.48 \\
\hline $\mathrm{T}_{11}-100 \% \mathrm{NPK}+$ Lime & $\begin{array}{l}5.60 \\
(2.45)\end{array}$ & $\begin{array}{l}5.28 \\
(2.46)\end{array}$ & $\begin{array}{l}6.31 \\
(2.60)\end{array}$ & $\begin{array}{l}6.49 \\
(2.63)\end{array}$ & 5.92 \\
\hline $\mathrm{T}_{12^{-}}$Control & $\begin{array}{l}10.36 \\
(3.32)\end{array}$ & $\begin{array}{l}10.91 \\
(3.36)\end{array}$ & $\begin{array}{l}10.66 \\
(3.22)\end{array}$ & $\begin{array}{l}12.03 \\
(3.53)\end{array}$ & 10.91 \\
\hline $\mathrm{SE}(\mathrm{m}) \pm$ & 0.14 & 0.11 & 0.13 & 0.06 & - \\
\hline $\mathrm{CD}(5 \%)$ & 0.41 & 0.33 & 0.37 & 0.19 & - \\
\hline $\mathrm{CV}(\%)$ & 10.45 & 8.35 & 9.34 & 4.45 & - \\
\hline
\end{tabular}

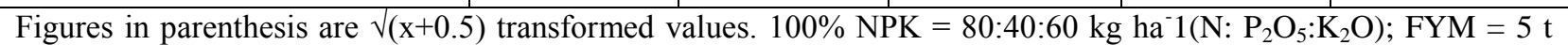
$\mathrm{ha}^{-1 ;}$ Lime $=1 \mathrm{t} \mathrm{ha}^{-1 ;} \mathrm{Zn}=12.5 \mathrm{~kg} \mathrm{Zn} \mathrm{SO}_{4} \mathrm{ha}^{-1 ;} \mathrm{B}=0.25 \% ; \mathrm{S}=30 \mathrm{~kg}_{\text {gypsum ha }}{ }^{-1}$. 
Table.5 Incidence of whorl maggot in rice during summer 2014- 2015, at Bhubaneswar

\begin{tabular}{|c|c|c|c|c|}
\hline \multirow[t]{2}{*}{ Treatments } & \multicolumn{3}{|c|}{ leaf infestation (\%) at } & \multirow{2}{*}{$\begin{array}{c}\text { Mean (\%) } \\
\text { leaf damage }\end{array}$} \\
\hline & 30 DAT & 40DAT & 50DAT & \\
\hline $\mathrm{T}_{1^{-}}-100 \% \mathrm{PK}$ & $\begin{array}{l}1.66 \\
(1.46)\end{array}$ & $\begin{array}{l}1.96 \\
(1.56)\end{array}$ & $\begin{array}{l}2.29 \\
(1.65)\end{array}$ & 1.97 \\
\hline $\mathrm{T} \mathrm{T}_{2}-100 \% \mathrm{NPK}$ & $\begin{array}{l}1.72 \\
(1.48)\end{array}$ & $\begin{array}{l}1.82 \\
(1.51)\end{array}$ & $\begin{array}{l}2.35 \\
(1.64)\end{array}$ & 1.96 \\
\hline $\mathrm{T}_{3}-150 \% \mathrm{NPK}$ & $\begin{array}{l}1.82 \\
(1.51)\end{array}$ & $\begin{array}{l}1.90 \\
(1.54)\end{array}$ & $\begin{array}{l}2.22 \\
(1.64)\end{array}$ & 1.98 \\
\hline $\mathrm{T}_{4}-100 \% \mathrm{NPK}+\mathrm{Zn}$ & $\begin{array}{l}1.54 \\
(1.42)\end{array}$ & $\begin{array}{l}1.68 \\
(1.46)\end{array}$ & $\begin{array}{l}1.70 \\
(1.48)\end{array}$ & 1.64 \\
\hline $\mathrm{T}_{5}-100 \% \mathrm{NPK}+\mathrm{FYM}$ & $\begin{array}{l}1.66 \\
(1.46)\end{array}$ & $\begin{array}{l}1.77 \\
(1.50)\end{array}$ & $\begin{array}{l}1.88 \\
(1.54)\end{array}$ & 1.77 \\
\hline $\begin{array}{l}\mathrm{T}_{6}-100 \% \\
\text { Lime }\end{array}$ & $\begin{array}{l}1.61 \\
(1.43)\end{array}$ & $\begin{array}{l}1.70 \\
(1.47)\end{array}$ & $\begin{array}{l}1.73 \\
(1.49)\end{array}$ & 1.68 \\
\hline $\mathrm{T}_{7}-100 \% \mathrm{NPK}+\mathrm{B}+\mathrm{Zn}$ & $\begin{array}{l}1.52 \\
(1.41)\end{array}$ & $\begin{array}{l}1.62 \\
(1.45)\end{array}$ & $\begin{array}{l}1.67 \\
(1.47)\end{array}$ & 1.60 \\
\hline $\mathrm{T}_{8^{-}} 100 \% \mathrm{NPK}+\mathrm{S}+\mathrm{Zn}$ & $\begin{array}{l}1.60 \\
(1.44)\end{array}$ & $\begin{array}{l}1.67 \\
(1.47)\end{array}$ & $\begin{array}{l}1.71 \\
(1.48)\end{array}$ & 1.66 \\
\hline $\mathrm{T}_{9}-100 \% \mathrm{~N}$ & $\begin{array}{l}2.04 \\
(1.59)\end{array}$ & $\begin{array}{l}2.15 \\
(1.62)\end{array}$ & $\begin{array}{l}2.35 \\
(1.69)\end{array}$ & 2.18 \\
\hline $\mathrm{T}_{10}-100 \% \mathrm{NP}$ & $\begin{array}{l}2.19 \\
(1.63)\end{array}$ & $\begin{array}{l}2.31 \\
(1.67)\end{array}$ & $\begin{array}{l}2.40 \\
(1.72)\end{array}$ & 2.30 \\
\hline $\mathrm{T}_{11}-100 \% \mathrm{NPK}+$ Lime & $\begin{array}{l}1.63 \\
(1.46)\end{array}$ & $\begin{array}{l}1.72 \\
(1.48)\end{array}$ & $\begin{array}{l}1.85 \\
(1.52)\end{array}$ & 1.73 \\
\hline $\mathrm{T}_{12^{-}}$Control & $\begin{array}{l}2.27 \\
(1.66)\end{array}$ & $\begin{array}{l}2.41 \\
(1.70)\end{array}$ & $\begin{array}{l}2.50 \\
(1.69)\end{array}$ & 2.39 \\
\hline $\mathrm{SE}(\mathrm{m}) \pm$ & 0.02 & 0.02 & 0.02 & - \\
\hline $\mathrm{CD}(5 \%)$ & 0.07 & 0.05 & 0.07 & - \\
\hline $\mathrm{CV}(\%)$ & 3.10 & 2.14 & 3.09 & - \\
\hline
\end{tabular}

Figures in parenthesis are $\sqrt{ }(\mathrm{x}+0.5)$ transformed values. $100 \% \mathrm{NPK}=80: 40: 60 \mathrm{~kg}$ ha $1\left(\mathrm{~N}^{-} \mathrm{P}_{2} \mathrm{O}_{5}: \mathrm{K}_{2} \mathrm{O}\right) ; \mathrm{FYM}=5 \mathrm{t}$ $\mathrm{ha}^{-1 ;}$ Lime $=1 \mathrm{t} \mathrm{ha}^{-1 ;} \mathrm{Zn}=12.5 \mathrm{~kg} \mathrm{Zn} \mathrm{SO}_{4} \mathrm{ha}^{-1 ;} \mathrm{B}=0.25 \% ; \mathrm{S}=30 \mathrm{~kg}_{\text {gypsum ha }}{ }^{-1}$. 
Table.6 Incidence of whorl maggot in rice during kharif - 2015, at Bhubaneswar

\begin{tabular}{|c|c|c|c|c|}
\hline \multirow[t]{2}{*}{ Treatments } & \multicolumn{3}{|c|}{ leaf infestation (\%) at } & \multirow{2}{*}{$\begin{array}{c}\text { Mean (\%) } \\
\text { leaf damage }\end{array}$} \\
\hline & 30 DAT & 40DAT & 50DAT & \\
\hline $\mathrm{T}_{1-}-100 \% \mathrm{PK}$ & $\begin{array}{l}0.80 \\
(1.13)\end{array}$ & $\begin{array}{l}1.17 \\
(1.29)\end{array}$ & $\begin{array}{l}1.17 \\
(1.29)\end{array}$ & 1.04 \\
\hline $\mathrm{T} \mathrm{T}_{2}-100 \% \mathrm{NPK}$ & $\begin{array}{l}1.42 \\
(1.37)\end{array}$ & $\begin{array}{l}1.42 \\
(1.38)\end{array}$ & $\begin{array}{l}1.67 \\
(1.46)\end{array}$ & 1.50 \\
\hline $\mathrm{T}_{3}-150 \% \mathrm{NPK}$ & $\begin{array}{l}1.55 \\
(1.42)\end{array}$ & $\begin{array}{l}1.67 \\
(1.46)\end{array}$ & $\begin{array}{l}1.82 \\
(1.51)\end{array}$ & 1.68 \\
\hline $\mathrm{T}_{4}-100 \% \mathrm{NPK}+\mathrm{Zn}$ & $\begin{array}{l}1.35 \\
(1.35)\end{array}$ & $\begin{array}{l}1.50 \\
(1.41)\end{array}$ & $\begin{array}{l}1.57 \\
(1.43)\end{array}$ & 1.47 \\
\hline $\mathrm{T}_{5}-100 \% \mathrm{NPK}+\mathrm{FYM}$ & $\begin{array}{l}0.71 \\
(1.09)\end{array}$ & $\begin{array}{l}1.00 \\
(1.31)\end{array}$ & $\begin{array}{l}1.17 \\
(1.29)\end{array}$ & 0.96 \\
\hline $\mathrm{T}_{6}-100 \% \mathrm{NPK}+\mathrm{FYM}+$ Lime & $\begin{array}{l}0.72 \\
(1.10)\end{array}$ & $\begin{array}{l}1.20 \\
(1.31)\end{array}$ & $\begin{array}{l}1.22 \\
(1.31)\end{array}$ & 1.04 \\
\hline $\mathrm{T}_{7}-100 \% \mathrm{NPK}+\mathrm{B}+\mathrm{Zn}$ & $\begin{array}{l}0.92 \\
(1.19)\end{array}$ & $\begin{array}{l}1.30 \\
(1.33)\end{array}$ & $\begin{array}{l}1.30 \\
(1.36)\end{array}$ & 1.17 \\
\hline $\mathrm{T}_{8}-100 \% \mathrm{NPK}+\mathrm{S}+\mathrm{Zn}$ & $\begin{array}{l}1.17 \\
(1.26)\end{array}$ & $\begin{array}{l}1.35 \\
(1.35)\end{array}$ & $\begin{array}{l}1.42 \\
(1.38)\end{array}$ & 1.31 \\
\hline $\mathrm{T}_{9}-100 \% \mathrm{~N}$ & $\begin{array}{l}1.65 \\
(1.52)\end{array}$ & $\begin{array}{l}1.72 \\
(1.48)\end{array}$ & $\begin{array}{l}2.15 \\
(1.62)\end{array}$ & 1.84 \\
\hline $\mathrm{T}_{10}-100 \% \mathrm{NP}$ & $\begin{array}{l}1.77 \\
(1.50)\end{array}$ & $\begin{array}{l}2.05 \\
(1.58)\end{array}$ & $\begin{array}{l}2.33 \\
(1.67)\end{array}$ & 2.05 \\
\hline $\mathrm{T}_{11}-100 \% \mathrm{NPK}+$ Lime & $\begin{array}{l}1.45 \\
(1.40)\end{array}$ & $\begin{array}{l}1.55 \\
(1.42)\end{array}$ & $\begin{array}{l}1.72 \\
(1.48)\end{array}$ & 1.57 \\
\hline $\mathrm{T}_{12^{-}}$Control & $\begin{array}{l}0.80 \\
(1.13)\end{array}$ & $\begin{array}{l}1.17 \\
(1.26)\end{array}$ & $\begin{array}{l}1.05 \\
(1.24)\end{array}$ & 1.00 \\
\hline $\mathrm{SE}(\mathrm{m}) \pm$ & 0.05 & 0.05 & 0.03 & - \\
\hline $\mathrm{CD}(5 \%)$ & 0.13 & 0.13 & 0.10 & - \\
\hline $\mathrm{CV}(\%)$ & 7.21 & 6.61 & 4.89 & - \\
\hline
\end{tabular}

Figures in parenthesis are $\sqrt{ }(\mathrm{x}+0.5)$ transformed values. $100 \% \mathrm{NPK}=80: 40: 60 \mathrm{~kg}$ ha ${ }^{-1}\left(\mathrm{~N}: \mathrm{P}_{2} \mathrm{O}_{5}: \mathrm{K}_{2} \mathrm{O}\right) ; \mathrm{FYM}=5 \mathrm{t}$ $\mathrm{ha}^{-1 ;}$ Lime $=1 \mathrm{tha}^{-1 ;} \mathrm{Zn}=12.5 \mathrm{~kg} \mathrm{Zn} \mathrm{SO}_{4} \mathrm{ha}^{-1 ;} \mathrm{B}=0.25 \% ; \mathrm{S}=30 \mathrm{~kg}_{\text {gypsum ha }}{ }^{-1}$. 


\section{Effect of fertilizers, fertilizer combinations} and manure on incidence of whorl maggot

The data on incidence of whorl maggot during summer 2014-15 is presented in table 5. It was observed that (\%) leaf damage was lowest in $\mathrm{T}_{7}(100 \% \mathrm{NPK}+\mathrm{B}+\mathrm{Zn})$ followed by $\mathrm{T}_{4}(100 \% \mathrm{NPK}+\mathrm{Zn}), \mathrm{T}_{8}(100 \% \mathrm{NPK}+\mathrm{S}+$ $\mathrm{Zn})$ and $\mathrm{T}_{6}(100 \% \mathrm{NPK}+\mathrm{FYM}+$ Lime $)$ than the rest of treatments. Thus, it clearly indicated that either individual nutrient or combination of nutrients i.e. NPK produced more $(\%)$ leaf damage as compared to $100 \%$ NPK supplemented with either B, Zn, S and FYM. During kharif- 2015 there was a change in trend as $\mathrm{T}_{5}(100 \% \mathrm{NPK}+\mathrm{FYM})$ produced $0.96 \%$ leaf damage followed by $\mathrm{T}_{6}(100 \%$ $\mathrm{NPK}+\mathrm{FYM}+$ Lime) with $1.04 \%$ leaf damage, $\mathrm{T}_{1}(100 \% \mathrm{PK})$ with $1.04 \%$ leaf damage and $\mathrm{T}_{7}(100 \% \mathrm{NPK}+\mathrm{B}+\mathrm{Zn})$ with $1.17 \%$ leaf damage (Table 6). Thus, treatments comprising of $100 \%$ NPK with supplements like Zn, FYM etc reduced the incidence of $(\%)$ leaf damage as compared to rest of treatments. There is no record available in literature to substantiate the present finding could be traced in literature.

The study has demonstrated that individual nutrient or combination of nutrients i.e. NPK produced more pest incidence compared to application of nutrients (NPK) along with combination of micronutrients had decreased the incidence of yellow borer, leaf folder and whorl maggot. So judicious use NPK fertilizers is considered as vital cultural practices in the IPM strategy of rice which minimise the insect pest incidence, markedly increase the yield and improve the quality of rice.

\section{Acknowledgements}

Authors acknowledge the OUAT for the support to undertake this study through its Long Term Fertilizer Effect (LTFE) project (AICRP).

\section{References}

Barbour, J.D., Farrar, R. R. and Kennedy, G. G. 1991. Interaction of fertilizer regime with host plant resistant in tomato. Entomology Experimental Applied, 60 (2): 289-300.

Chakraborty Kaushik, and Debes Chandra Deb. 2011. Impact of proportional application of organic and inorganic fertilizers on the incidence of paddy yellow stem borer, Scircophaga incertulas Walker population during kharifseason. Wesleyan Journal of Research, 4(1).

Chau Luong Minh, and Heong K L. 2005. Effects of organic fertilizers on insect pest and diseases of rice. Omonrice, 13: 26-33.

Dash, D., Mishra P R and Panigrahi D. 2008. Effect of plant nutrients on the incidence of yellow stem borer. Oryza, 45(45): 333-335.

Dash, D., Senapati, B and Patnaik, H. P. 2011. Influence of rice varieties and plant nutrients on the incidence of yellow stem borer, Scirpophaga incertulas (walker) under protected condition. Indian Journal of Entomology, 73(3):96-200.

Gomez, K.A., 1972. Measuring stem borer incidence. In: Techniques for field experiments with rice. Ahandbook. Int. Rice Res. Institute, Los Bonos, Laguna, Phillipines, Pp.39-40

Hyrsl, P., Buyukguzel E and Buyukguzel K. 2007. The effects of boric acid induced oxidative stress on antioxidant enzymes and survivorship in Galleria mellonella. Insect Biochem, 66: 23-31.

Khush, G.S., 2005. What it will take to feed five billion rice consumers by 2030. Plant Molecular Biology, 59: 1-6.

Lal, O.P., 1996. Recent advances in Entomology (Ed.) Lal, O.P. APC publications Pvt. Ltd., New Delhi, pp.392

Panda, N., 1976. Role of chelated boron and 
zinc in host plantresistance of rice to yellow rice borer, Tryporyza incertulas walker. Proceedings, 63rd Indian Science Congress, 111:48

Panda, N., Mohapatra, S., Bhaduari, S. and Das, R.C. 1975. Effect of Chelating agents on the resistance of Brinjal plants to Leucinodes orbonalis Guen. South Indian Horticulture, 23, 141-144

Premila, K.S., and Dale D. 1984. Induction of resistance in rice plants to insect pests by the application of chelated metal complexes. Crop Protection 3(2):187192.

Samiayyan, K., and Janarthanan, R. 1988 b. Effect of potassium in combination with nitrogen on the incidence of green leaf hopper in rice. Plant Protection Bulletin, 40(2): 1-3.
Sarwa Muhammad, 2011. Effects of Zinc fertilizer application on the incidence of rice stem borers (Scirpophaga species) (Lepidoptera: Pyralidae) in rice (Oryza sativa L.) crop. Journal of Cereals and Oilseeds, 2(5): 61-65.

Shu, Y. H., Gao, Y.Y.; Sun, H. X.; Zou, Z. W.; Zhou, Q. A.; Zhang, G. R. (2009). Effects of Zinc exposure on the reproduction of Spodoptera litura Fabricius (Lepidoptera: Noctuidae). Ecotoxicol. Environ. Saf. 72(8): 2130-2136.

Yarasi, B., Sadumpati, V.; Immanni, C. P.; Vudem, D. R. and Khareedu, V. R. 2008. Transgenic rice expressing Allium sativum leaf agglutinin (ASAL) exhibits high-level resistance against major sapsucking pests. Plant Biology, 8: 102-115.

\section{How to cite this article:}

Madhuri, G., P.C. Dash and Pruthvi, P. 2017. Impact of Different Graded Levels of Fertilizers on the Incidence of Yellow Stem Borer Scirpophaga incertulas (Wlk.), Leaf Folder Cnaphalocrocis medinalis (Guenee) and Whorl Maggot Hydrellia philippina (Ferino) in Paddy. Int.J.Curr.Microbiol.App.Sci. 6(9): 793-803. doi: https://doi.org/10.20546/ijcmas.2017.609.098 